\title{
Phylogenetic analysis of cultivable oral treponemes from the Smibert collection
}

\author{
B. J. Paster, F. E. Dewhirst, B. C. Coleman, C. N. Lau and R. L. Ericson \\ Author for correspondence: B. J. Paster. Tel: +16172625200 ext. 288. Fax: +16172624021 \\ e-mail: bpaster@forsyth.org
}

Department of Molecular Genetics, Forsyth Dental Center, 140 Fenway, Boston, MA 02115, USA
Dr Robert Smibert from the Virginia Polytechnic Institute, USA, isolated and collected over 200 strains of oral treponemes over a 20-year period. Dr Smibert, Dr W. E. C. Moore and Dr L. V. Moore separated these isolates and reference strains into different groups on the basis of cellular fatty acid analysis. In this study, the 16S rRNA genes were sequenced for 47 strains that were representative of these groups. Five distinct species were identified on the basis of 165 rRNA sequence comparisons; two of these species are newly named and three have not yet been characterized. The first species, designated Treponema Smibert-1, was represented by the single strain D4B-1 and was later identified as the newly described Treponema maltophilum. However, strain D4B-1 possessed a different flagellar arrangement to that of $T$. maltophilum. The second species, Treponema Smibert-2, was represented by nine isolates that possessed identical 16S rRNA gene sequences. The closest relatives of this species were Treponema Smibert-3 and Treponema Smibert-4 at approximately $90 \%$ sequence similarity. Within Treponema Smibert-2, there was no correlation between phylogenetic analysis and cellular fatty acid analysis since six different cellular fatty acid groups represented the nine strains. Treponema Smibert-3 (strain D36ER-1) and Treponema Smibert-4 (D62CR-12) were each represented by only a single strain and were closely related to each other at $98 \%$ sequence similarity. Strain D36ER-1 of Treponema Smibert-3 was identified as belonging to the not-yet-cultivated phylotype 20 [Choi, B. K., Paster, B. J., Dewhirst, F. E. \& Göbel, U. B. (1994). Infect Immun 62,1889-1895]. Strain D62CR-12 of Treponema Smibert-4 was nearly identical in sequence to the newly described Treponema amylovorum. The fifth species, Treponema Smibert-5, was represented by a single strain, D120CR-1, and was closely related at about $98 \%$ sequence similarity to the three subspecies of Treponema socranskii. The phylogenetic analyses of strains of Treponema vincentii and of subspecies of $T$. socranskii are also reported. The closest oral relatives of $T$. vincentii were Treponema medium at $98.7 \%$ sequence similarity and Treponema denticola at $\mathbf{9 1 . 5} \%$ sequence similarity. $T$. socranskii subspp. socranskii, buccale and paredis formed three separate phylogenetic branches with sequence similarities of about $98 \%$ to each other. The closest relative of the subspecies of $T$. socranskii and of Smibert-5 was Smibert-2 at about $86 \%$ sequence similarity. Historic reference strains Fuji, ' Treponema ambigua', Fm, Ichelson-2, N-39, TD2, TRRD, MRB, IPP, Jethro and T32A, as well as an unknown strain designated only as Treponema oralis, were identified as strains of $T$. denticola. Reference strains Fuji, Jethro, T32A and IPP plus three isolates of the Smibert collection were also contaminated with a mycoplasma as determined by 165 rRNA comparative analysis. Consequently, spirochaetal cultures should be screened for mycoplasmas. There are presently at least ten species of cultivable oral species of Treponema with the cut-off for separate species 


\section{designation at about $98 \%$ sequence similarity. However, DNA-DNA reassociation experiments are necessary to differentiate species when 165 rDNA sequence similarities are at about this level.}

Keywords: phylogeny, 16S rDNA, oral spirochaete, Treponema

\section{INTRODUCTION}

It has been reported from microscopic examinations that oral treponemes can represent up to $50 \%$ of the detectable flora in subgingival plaque from diseased sites $(1,23)$. Spirochaetes are present in elevated numbers in subgingival plaque of subjects with a variety of oral diseases that include gingivitis $(19,25)$, acute necrotizing ulcerative gingivitis (ANUG) $(8,14$, 18 ), juvenile periodontitis $(3,28)$, chronic periodontitis $(20,21)$, rapidly progressing periodontitis $(27,29,38)$, periocoronitis (6) and endodontic abscesses (43). HIVpositive subjects with gingivitis and adult periodontitis also have elevated numbers of spirochaetes $(26,35)$. In sites of ANUG, juvenile periodontitis and advanced periodontal disease, spirochaetes have been observed invading the connective tissue or intact epithelium (3, $11,18,33,34,36)$. In some instances, nearly confluent monolayers of spirochaetes have been observed adhering to the epithelial lining of inflamed periodontal pockets $(16,18,19)$.

By comparison of $16 \mathrm{~S}$ rRNA or $16 \mathrm{~S}$ rDNA sequences, spirochaetes are divided into two major phylogenetic groupings $(7,13,31)$. The first grouping, which corresponds to the family Spirochaetaceae, contains species of the genera Treponema, Spirochaeta, Borrelia, Serpulina (and Brachyspira) and Brevinema. However, a few species of Spirochaeta are more closely related to the treponemes than to other members of the genus Spirocheata (31). The second grouping, which corresponds to the family Leptospiraceae, contains species of the genera Leptospira and Leptonema. Thus far, spirochaetes isolated from the oral cavity all fall within the genus Treponema.

Although spirochaetes have been associated with oral diseases for many years, only seven species have been cultured and validly named: Treponema denticola (39), Treponema pectinovorum (40), Treponema socranskii (including three subspecies) (41), Treponema vincentii (39), and, more recently, Treponema maltophilum (48), Treponema medium (45) and Treponema amylovorum (49). Despite recent development of improved culture conditions and artificial media $(17,32,44,48)$, many of the spirochaetal types often observed microscopically, i.e. the 'medium to large' spirochaetes $(9,21,47)$, have still resisted efforts for long-term in vitro cultivation. However, over 20 species of not-yet-cultivated oral treponemes have been identified by sequencing cloned spirochaetal 16S rRNA genes that were amplified from plaque DNA from a patient with severe periodontitis (5). In addition, species of not-yet-cultivated trepo- nemes from digital dermatitis lesions in cattle have been shown to be related to some oral treponemes (4).

Robert Smibert (Virginia Polytechnic Institute, Blacksburg, VA, USA) isolated and collected oral spirochaetes over a 20-year period. The Smibert collection contains approximately 250 spirochaetal isolates including some reference strains. W. E. C. Moore and L. V. Moore (also of the Virginia Polytechnic Institute) classified the Smibert isolates into lettered groups (A through V) based upon their biochemical characteristics and cellular fatty acid profiles $(25,26,27,28,40)$. In this study, $16 \mathrm{~S}$ rRNA gene sequence comparisons were used to determine the phylogenetic identity of representatives of the cellular fatty acid groups and of other oral cultivable Treponema species not previously analysed, such as $T$. vincentii and subspecies of $T$. socranskii. Another goal was to determine the correlation between phylogenetic analysis and cellular fatty acid analysis of oral treponemes.

A preliminary report of these results was recently published (12).

\section{METHODS}

Bacterial strains. Dr W. E. C. Moore and Dr L. V. Moore of Virginia Polytechnic Institute provided strains to us and to ATCC as frozen cell pellets or suspensions. Strains analysed in this study are listed in Table 1. Cells were initially grown in NOS media supplemented with pectin (40). Cultures were incubated in an anaerobic environment at $37^{\circ} \mathrm{C}$ for $3-5 \mathrm{~d}$. In this study, cells from the frozen samples were used directly for analysis. Strains are available from ATCC, although specific accession numbers have not yet been assigned.

DNA isolation. Approximately $1-5 \times 10^{7}$ cells from frozen cell samples were suspended in lysis buffer $(50 \mathrm{mM}$ Tris $/ \mathrm{HCl}$ $\mathrm{pH} 7 \cdot 6,1 \mathrm{mM}$ EDTA, $0 \cdot 5 \%$ Tween $20,200 \mu \mathrm{g}$ proteinase $\mathrm{K}$ $\mathrm{ml}^{-1}$ ) and incubated at $55^{\circ} \mathrm{C}$ for $2 \mathrm{~h}$. The proteinase $\mathrm{K}$ was subsequently inactivated by heating at $95^{\circ} \mathrm{C}$ for $10 \mathrm{~min}$. Alternatively, $5 \mu \mathrm{l}$ of a suspension containing $10^{8}$ cells $\mathrm{ml}^{-1}$ was treated with $15 \mu \mathrm{l}$ GeneReleaser (Bioventures) and microwaved on high for $5 \mathrm{~min}$. Aliquots of the supernatant were used for the DNA template.

Amplification of 165 rRNA cistrons. Universally conserved primers [primers C70 and B37 (10)] were used to amplify $16 \mathrm{~S}$ rRNA genes. A spirochaetal selective reverse primer, $\mathrm{C} 90\left(5^{\prime}\right.$ GTT ACG ACT TCA CCC TCC T $3^{\prime}$ ), was used with a universal forward primer, C75 (5' GAG AGT TTG ATY CTG GCT CAG 3'), if the universal primers did not produce PCR product. PCR reactions were performed in thin-walled tubes using a Perkin-Elmer 480 thermal cycler. Three microlitres of the crude DNA and $1 \mu \mathrm{M}$ primers were added to the reaction mixture (as suggested by the manufacturer), 
Table 1. Sources and accession numbers of the strains sequenced

\begin{tabular}{|c|c|c|}
\hline Bacterial species & Strain & $\begin{array}{c}\text { GenBank } \\
\text { accession no. }\end{array}$ \\
\hline \multicolumn{3}{|l|}{ Reference species } \\
\hline Spirochaeta caldaria & $\mathrm{H} 1^{\mathrm{T}}$ & M71240 \\
\hline Spirochaeta stenostrepta & ATCC $25083^{\mathrm{T}}$ & M34264 \\
\hline Spirochaeta zuelzerae & ATCC $19044^{\mathrm{T}}$ & M34265 \\
\hline Treponema amylovorum & $\mathrm{HA} 2 \mathrm{P}^{\mathrm{T}}$ & Y09959 \\
\hline Treponema bryantii & ATCC $33254^{\mathrm{T}}$ & M57737 \\
\hline Treponema denticola & ATCC 33520 & M71236 \\
\hline Treponema maltophilum & $\mathrm{BR}^{\mathrm{T}}$ & $\mathrm{X} 87140$ \\
\hline Treponema medium & $\mathrm{G} 7201^{\mathrm{T}}$ & D85437 \\
\hline Treponema pectinovorum & ATCC $33768^{\mathrm{T}}$ & M71237 \\
\hline Treponema pallidum & Nichols & M34266 \\
\hline Treponema phagedenis & K5 & M57739 \\
\hline Treponema saccharophilum & ATCC $43261^{\mathrm{T}}$ & M71238 \\
\hline Treponema sp. & CA & M59294 \\
\hline Treponema succinifaciens & ATCC $33096^{\mathrm{T}}$ & M57738 \\
\hline \multicolumn{3}{|l|}{ Species isolated in this study } \\
\hline $\begin{array}{l}\text { Treponema socranskii subsp. } \\
\text { socranskii }\end{array}$ & $\operatorname{ATCC} 35536^{\mathrm{T}}$ & AF033306 \\
\hline $\begin{array}{l}\text { Treponema socranskii subsp. } \\
\text { buccale }\end{array}$ & ATCC $35534^{\mathrm{T}}$ & AF033305 \\
\hline $\begin{array}{l}\text { Treponema socranskii subsp. } \\
\text { paredis }\end{array}$ & $\operatorname{ATCC} 35535^{\mathrm{T}}$ & AF033307 \\
\hline Treponema socranskii subsp. 04 & D11B-2 & AF033308 \\
\hline Treponema sp. Smibert-1 & D4B-1 & AF023039 \\
\hline Treponema sp. Smibert-2 & D96NR-3 & AF033304 \\
\hline Treponema sp. Smibert-3 & D36ER-1 & AF023044 \\
\hline Treponema sp. Smibert-4 & D62CR-I2 & AF023045 \\
\hline Treponema sp. Smibert-5 & D120CR-1 & AF033303 \\
\hline Treponema vincentii & N-9 (ATCC 700013) & AF033310 \\
\hline Treponema vincentii & ATCC 35580 & AF033309 \\
\hline
\end{tabular}

which had a final volume of $82 \mu \mathrm{l}$. Ampliwax PCR Gem 100s (PE Applied Biosystems) were used in a hot-start protocol. The following conditions were used for amplification using primers C70 and B37: denaturation at $94^{\circ} \mathrm{C}$ for $45 \mathrm{~s}$, annealing at $50^{\circ} \mathrm{C}$ for $45 \mathrm{~s}$ and elongation at $72^{\circ} \mathrm{C}$ for $90 \mathrm{~s}$ with an additional $5 \mathrm{~s}$ added for each cycle. A total of 30 cycles were performed followed by a final elongation step at $72{ }^{\circ} \mathrm{C}$ for $15 \mathrm{~min}$. Conditions for amplification using primers $\mathrm{C} 90$ and $\mathrm{C} 75$ were identical except that the annealing temperature was $60^{\circ} \mathrm{C}$. After removal of Ampliwax, 0.6 vol. $20 \%$ PEG 8000 (Sigma) in $2.5 \mathrm{M} \mathrm{NaCl}$ was added and the mixture incubated at $37^{\circ} \mathrm{C}$ for $10 \mathrm{~min}$ to precipitate the DNA. The sample was centrifuged for $15 \mathrm{~min}$ at $15000 \mathrm{~g}$ and the pellet was washed with $80 \%$ ethanol. The pellet was dissolved in $35 \mu \mathrm{l}$ sterile filtered water. For automated sequencing, amplified DNA was purified by washing on a QiaQuick PCR Purification Kit (Qiagen), following the manufacturer's protocol.

Sequencing methods. The DNA sample from PCR was directly sequenced using a TAQuence cycle-sequencing kit (USB) or a fmol DNA Sequencing System kit (Promega). Primers (10) were end-labelled with $\left[\gamma^{33}\right.$ P]ATP (NEN/ Dupont) using the manufacturer's protocol. Purified DNA (25-80 ng) from the PCR was used for each sequencing reaction. Reaction products were run electrophoretically onto $8 \%$ polyacrylamide/urea gels and subsequently detected by exposure of dried gels to X-ray film for 24-48 h. More recently, purified DNA from PCR was sequenced using an ABI prism cycle-sequencing kit (dRhodamine Terminator Cycle Sequencing kit with AmpliTaq DNA polymerase FS, Perkin-Elmer). The manufacturer's protocol was followed. Sequencing was performed using an ABI 377 DNA sequencer.

165 rRNA sequence analysis. Programs for data entry, editing, sequence alignment, secondary structure comparison, similarity matrix generation and phylogenetic tree construction were written in Microsoft QuickBASIC for use on IBM PC-AT and compatible computers (30). Over 5000 sequences from our database, the Ribosomal Database Project (22) and GenBank were available for sequence comparisons. Sequences of all known cultivable and not-yetcultivated species of Treponema have been deposited in GenBank. Similarity matrices were constructed from the aligned sequences by using only those sequence positions for which $90 \%$ of strains had data. The similarity matrices were corrected for multiple base changes by the method of Jukes $\&$ Cantor (15). Phylogenetic trees were constructed using the neighbour-joining method of Saitou \& Nei (37).

Electron microscopy. Cells were suspended in $10 \mathrm{mM}$ Tris 
Table 2. Phylogenetic and cellular fatty acid identity of sequenced strains from the Smibert collection

\begin{tabular}{|c|c|c|c|c|}
\hline Phylogenetic identity & $\begin{array}{l}\text { VPI or reference } \\
\text { strain no.* }\end{array}$ & $\begin{array}{l}\text { Cellular fatty acid } \\
\text { identity } \dagger\end{array}$ & Clinical data $\ddagger$ & Sequence§ \\
\hline $\begin{array}{l}\text { Treponema Smibert-1 } \\
\text { (T. maltophilum) }\end{array}$ & D4B-1 & $\mathrm{F}$ or $\mathrm{C}$ & Not available & Full \\
\hline \multirow[t]{9}{*}{ Treponema Smibert-2 } & D52CR-1 & $\mathrm{S}$ & Supragingival, severe & Full \\
\hline & D79TR-1 & F & $\begin{array}{l}\text { Subgingival, moderate, } \\
4 \mathrm{~mm}\end{array}$ & Partial \\
\hline & D79WRI1 & $\mathrm{U}$ & Subgingival, moderate & Partial \\
\hline & D80BRII-5 & $\begin{array}{l}\text { T. socranskii subsp. } \\
\text { socranskii }\end{array}$ & Subgingival, health & Full \\
\hline & D82MRI-1 & $\mathrm{E}$ & $\begin{array}{l}\text { Subgingival, moderate, } \\
5 \mathrm{~mm}\end{array}$ & Partial \\
\hline & D83PR 1 & $\mathrm{~T}$ & $\begin{array}{l}\text { Subgingival, moderate, } \\
6 \mathrm{~mm}\end{array}$ & Partial \\
\hline & D83RR-I1 & $\mathrm{F}$ & $\begin{array}{l}\text { Subgingival, moderate, } \\
3 \mathrm{~mm}\end{array}$ & Partial \\
\hline & D84AR-1 & $S$ & $\begin{array}{l}\text { Subgingival, moderate, } \\
8 \mathrm{~mm}\end{array}$ & Partial \\
\hline & D96NR-3 & F & $\begin{array}{l}\text { Subgingival, moderate, } \\
2 \mathrm{~mm}\end{array}$ & Full \\
\hline Treponema Smibert-3 & D36ER-1 & $\mathbf{F}$ & Supragingival, severe & Full \\
\hline $\begin{array}{l}\text { Treponema } \text { Smibert- } 4 \\
\text { (T. amylovorum) }\end{array}$ & D62CR-I2 & $\mathrm{N}$ & $\begin{array}{l}\text { Supragingival, JP, } \\
9 \mathrm{~mm}\end{array}$ & Full \\
\hline Treponema Smibert-5 & D120CR-1 & $\begin{array}{l}\text { T. socranskii subsp. } \\
\text { buccale }\end{array}$ & $\begin{array}{l}\text { Subgingival, health, } \\
3 \mathrm{~mm}\end{array}$ & Full \\
\hline \multirow[t]{16}{*}{ T. denticola } & D3A-1 & T. denticola & & Partial \\
\hline & D46DR-I1 & & & Partial \\
\hline & D64BPPI-1 & T. denticola & & Full \\
\hline & D79ZR-1 & $\mathrm{F}$ & & Partial \\
\hline & $\mathrm{Fm}$ & T. denticola & & Full \\
\hline & Fuji & T. denticola & & Partial \\
\hline & Ichelson 2 & T. denticola & & Full \\
\hline & IPP & T. denticola & & Partial \\
\hline & Jethro-monkey & T. denticola & & Partial \\
\hline & MRB & T. denticola & & Partial \\
\hline & $\mathrm{N}-39$ & T. denticola & & Partial \\
\hline & T. ambigua & $T$. denticola & & Partial \\
\hline & T. oralis & T. denticola & & \\
\hline & T32A & T. denticola & & Partial \\
\hline & TD2 & T. denticola & & Full \\
\hline & TRRD & T. denticola & & Partial \\
\hline T. pectinovorum & D48BR-1 & T. pectinovorum & & Partial \\
\hline \multirow{2}{*}{$\begin{array}{l}\text { T. socranskii subsp. } \\
\text { buccale }\end{array}$} & ATCC $35534^{\mathrm{T}}$ & T. socranskii subsp. & & Full \\
\hline & D64AR-5 & $\begin{array}{l}\text { buccale } \\
\mathrm{F}\end{array}$ & & Partial \\
\hline
\end{tabular}

(continued on facing page)

buffer ( $\mathrm{pH} 7 \cdot 4)$ at a concentration of approximately $10^{8}$ cells $\mathrm{ml}^{-1}$. Samples were negatively stained with $1 \%(\mathrm{w} / \mathrm{v})$ phosphotungstic acid (pH 6.6) for 20-30 s. Specimens were examined with a JEOL model JEM-1200EX transmission electron microscope operating at $100 \mathrm{kV}$.

\section{RESULTS AND DISCUSSION}

Dr W. E. C. Moore and Dr L. V. Moore divided the spirochaete strains in the Smibert collection into lettered groups $(\mathrm{A}-\mathrm{V})$ based upon their biochemical 
Table 2. (cont.)

\begin{tabular}{|c|c|c|c|c|}
\hline Phylogenetic identity & $\begin{array}{l}\text { VPI or reference } \\
\text { strain no.* }\end{array}$ & $\begin{array}{l}\text { Cellular fatty acid } \\
\text { identity }{ }^{\dagger}\end{array}$ & Clinical data $\$$ & Sequence $\S$ \\
\hline \multirow[t]{10}{*}{$\begin{array}{l}\text { T. socranskii subsp. } \\
\text { paredis }\end{array}$} & ATCC $35535^{\mathrm{T}}$ & $\begin{array}{l}\text { T. socranskii subsp. } \\
\text { paredis }\end{array}$ & & Full \\
\hline & D45APE-1 & $\mathrm{C}$ & & Partial \\
\hline & D46CPE-1 ${ }^{\mathrm{T}}$ 的 & $\begin{array}{l}\text { T. socranskii subsp. } \\
\text { paredis }\end{array}$ & & Partial \\
\hline & D45DPEI1 & $\mathrm{L}$ & & Full \\
\hline & D46CPE- 5 & $\mathrm{~L}$ & & Partial \\
\hline & D83BR1 & $\mathrm{L}$ & & Full \\
\hline & D130AR-1 & $\mathrm{V}$ & & Full \\
\hline & D151CRII-1 & $\begin{array}{l}\text { T. socranskii subsp. } \\
\text { paredis }\end{array}$ & & Full \\
\hline & E1H-3 & $\mathrm{C}$ & & Full \\
\hline & $\mathrm{E} 1 \mathrm{~F}-1$ & $\mathrm{E}$ & & Partial \\
\hline \multirow[t]{5}{*}{$\begin{array}{l}\text { T. socranskii subsp. } \\
\text { socranskii }\end{array}$} & ATCC $35536^{\mathrm{T}}$ & $\begin{array}{l}\text { T. socranskii subsp. } \\
\text { socranskii }\end{array}$ & & Full \\
\hline & D46DR-I3 & $\mathrm{N}$ & & Full \\
\hline & D50AR-I1a & T. socranskii subsp. 04 & & Partial \\
\hline & D84BR-1 & $\mathrm{G}$ & & Partial \\
\hline & D97RR-4 & Not available & & Partial \\
\hline \multirow[t]{5}{*}{$\begin{array}{l}\text { T. socranskii subsp. } \\
04\end{array}$} & D09B-1 & $\begin{array}{l}\text { T. socranskii subsp. } \\
04\end{array}$ & & Partial \\
\hline & D11B2 & $\begin{array}{l}\text { T. socranskii subsp. } \\
04\end{array}$ & & Full \\
\hline & D36FPE-1 & $\begin{array}{l}\text { T. socranskii subsp. } \\
04\end{array}$ & & Partial \\
\hline & D83DR-5 & $\begin{array}{l}\text { T. socranskii subsp. } \\
04\end{array}$ & & Partial \\
\hline & D108AR-4 & $\begin{array}{l}\text { T. socranskii subsp. } \\
04\end{array}$ & & Partial \\
\hline \multirow{9}{*}{$\begin{array}{l}\text { T. socranskii } \\
\text { unknown subspecies }\end{array}$} & D08B-1 & $\mathrm{C}$ & & Partial \\
\hline & D75BR-I3 & G & & Full \\
\hline & D75BR-III7 & $\mathrm{F}$ & & Full \\
\hline & D75BR-III8 & G & & Partial \\
\hline & D77DR-5 & $\mathrm{E}$ & & Partial \\
\hline & D79VR-1 & $\mathrm{F}$ & & Partial \\
\hline & D116CR-1 & $\mathrm{V}$ & & Partial \\
\hline & D135AR-I1 & DB & & Partial \\
\hline & E2D-II2 & $\mathrm{E}$ & & Partial \\
\hline$T$. vincentii & $\mathrm{N}-9$ & $T$. vincentii & & Full \\
\hline
\end{tabular}

*VPI strains begin with letters $\mathrm{D}$ or $\mathrm{E}$ followed by a patient number. The letter after the number represents the visit number; for example $\mathrm{C}$ is the third visit by that patient. The following suffixes after the patient ID are as follows: PE, isolated from pectin agar medium; $\mathbf{R}$, isolated from pectin agar medium supplemented with rifampicin and polymyxin; PP, isolated from peptone/yeast extract/glucose agar medium; I, colony type; I1, a single isolate from that colony type; I1a, a re-isolation of that particular strain. $\dagger$ Data from R. M. Smibert, Virginia Polytechnic Institute, VA, USA. Cellular fatty acid groups are designated as letters or known species. $\ddagger$ Data from R. M. Smibert, Virginia Polytechnic Institute, VA, USA. Clinical data, where known, are reported only for new species described in this study. Subgingival, subgingival plaque; supragingival, supragingival plaque; moderate, moderate adult periodontitis; severe, severe adult periodontitis; JP, juvenile periodontitis; health, healthy sites. Pocket depths, where known, are indicated by numbers. $\S$ Full indicates that a nearly complete $16 \mathrm{~S}$ rRNA sequence (approximately 1450 bases) of the strain was determined. Partial indicates that approximately $350-500$ bases were determined.

I Strain contaminated with a mycoplasma as determined by sequence analysis. Only mycoplasma sequences were obtained from strains D113DR-1 and D156DR-4. 
Table 3. Similarity matrix for the spirochaetal strains constructed from 16S rRNA sequences

Numbers above the diagonal represent percentages of similarity and those below the diagonal are percentages of difference corrected for multiple base changes.

\begin{tabular}{|c|c|c|c|c|c|c|c|c|c|c|c|c|c|c|c|c|c|c|c|c|c|c|c|c|}
\hline Bacterial species & $T d e$ & $T p h$ & $T p a$ & $T v i$ & Tme & $S z u$ & Ste & $S c a$ & Sm-1 & Tma & $T b r$ & $T p e$ & $T s a$ & $T s u$ & CA & Sm-2 & $\mathrm{Sm-3}$ & $\mathrm{Sm}-4$ & Tam & $S m-5$ & $T s b$ & $T_{s s}$ & $T s p$ & 704 \\
\hline Treponema denticola & - & $93 \cdot 1$ & 88.7 & 91.5 & 91.5 & $91 \cdot 1$ & $86 \cdot 7$ & 87.6 & 87.5 & $87 \cdot 4$ & 86.0 & $85 \cdot 9$ & $84 \cdot 2$ & $81 \cdot 3$ & $84 \cdot 0$ & 86.6 & $84 \cdot 1$ & $84 \cdot 8$ & $84 \cdot 7$ & 83.9 & $84 \cdot 0$ & 83.9 & 83.4 & $84 \cdot 4$ \\
\hline Treponema phagedenis & $7 \cdot 3$ & - & $90 \cdot 4$ & 92.0 & 91.8 & $91 \cdot 4$ & 87.6 & $87 \cdot 0$ & 86.7 & & $85 \cdot 2$ & $86 \cdot 0$ & $84 \cdot 0$ & 81.8 & 83.6 & $86 \cdot 3$ & & $85 \cdot 2$ & $85 \cdot 0$ & $84 \cdot 3$ & $84 \cdot 1$ & 3.7 & $83 \cdot 6$ & $84 \cdot 3$ \\
\hline Treponema pallidum & $12 \cdot 2$ & $10 \cdot 2$ & - & 88.4 & $88 \cdot 2$ & $88 \cdot 0$ & 86.0 & $86 \cdot 2$ & 84.5 & $84 \cdot 5$ & $83 \cdot 4$ & 83.6 & $82 \cdot 3$ & 81.8 & $83 \cdot 6$ & $84 \cdot 4$ & $83 \cdot 6$ & $83 \cdot 2$ & $83 \cdot 1$ & $82 \cdot 7$ & $82 \cdot 3$ & $82 \cdot 3$ & $81 \cdot 9$ & $82 \cdot 9$ \\
\hline Treponema vincentii & $9 \cdot 0$ & 8.4 & $12 \cdot 6$ & - & 98.7 & $90 \cdot 9$ & 87.2 & 87.8 & $87 \cdot 3$ & $87 \cdot 3$ & $85 \cdot 4$ & $85 \cdot 9$ & $83 \cdot 6$ & 80.7 & 83.5 & $86 \cdot 2$ & $84 \cdot 2$ & $84 \cdot 3$ & $84 \cdot 2$ & $83 \cdot 5$ & $83 \cdot 2$ & $83 \cdot 2$ & 82.7 & $83 \cdot 3$ \\
\hline Treponema medium & $9 \cdot 0$ & 8.7 & 12.8 & $1 \cdot 3$ & - & $91 \cdot 0$ & $87 \cdot 2$ & $88 \cdot 2$ & 87.5 & 87.5 & $85 \cdot 2$ & $85 \cdot 4$ & 83.7 & $80 \cdot 6$ & $83 \cdot 5$ & 85.8 & 83.8 & $84 \cdot 0$ & $84 \cdot 1$ & $83 \cdot 6$ & $83 \cdot 2$ & $83 \cdot 0$ & 82.7 & $83 \cdot 2$ \\
\hline Spirochaeta zuelzerae & 9.5 & $9 \cdot 1$ & $13 \cdot 1$ & $9 \cdot 7$ & $9 \cdot 6$ & - & $88 \cdot 7$ & 88.9 & 87.9 & 87.9 & $86 \cdot 5$ & $86 \cdot 3$ & $84 \cdot 7$ & $82 \cdot 4$ & $84 \cdot 4$ & 86.7 & $84 \cdot 7$ & $84 \cdot 9$ & $84 \cdot 9$ & $83 \cdot 6$ & $83 \cdot 2$ & $83 \cdot 2$ & $82 \cdot 9$ & $83 \cdot 5$ \\
\hline Spirochaeta stenostrepta & 14.6 & $13 \cdot 6$ & $15 \cdot 5$ & $14 \cdot 0$ & $14 \cdot 0$ & $12 \cdot 3$ & - & $94 \cdot 2$ & $84 \cdot 7$ & $84 \cdot 7$ & 85.4 & $84 \cdot 4$ & $82 \cdot 9$ & $82 \cdot 1$ & $85 \cdot 5$ & $84 \cdot 4$ & $84 \cdot 6$ & $84 \cdot 1$ & $84 \cdot 1$ & $83 \cdot 8$ & $83 \cdot 5$ & $83 \cdot 4$ & $83 \cdot 3$ & $84 \cdot 0$ \\
\hline Spirochaeta caldaria & $13 \cdot 5$ & $14 \cdot 2$ & $15 \cdot 2$ & $13 \cdot 3$ & $12 \cdot 8$ & $12 \cdot 0$ & $6 \cdot 1$ & - & 86.0 & $85 \cdot 8$ & 86.4 & $85 \cdot 4$ & $83 \cdot 8$ & 82.5 & $85 \cdot 2$ & $84 \cdot 6$ & $84 \cdot 4$ & $84 \cdot 3$ & $84 \cdot 3$ & $4 \cdot 4$ & $84 \cdot 1$ & 84.0 & $84 \cdot 1$ & $84 \cdot 0$ \\
\hline Treponema Smibert-1 & 13.7 & 14.7 & $17 \cdot 4$ & $13 \cdot 9$ & 13.7 & $13 \cdot 2$ & $17 \cdot 1$ & $15 \cdot 5$ & - & 99.8 & $86 \cdot 9$ & 87.8 & $82 \cdot 8$ & $81 \cdot 7$ & $84 \cdot 2$ & 87.6 & $84 \cdot 7$ & 85.6 & $85 \cdot 5$ & $4 \cdot 2$ & $84 \cdot 3$ & $84 \cdot 1$ & $84 \cdot 1$ & 84.0 \\
\hline Treponema maltophilum & 13.8 & $14 \cdot 4$ & $17 \cdot 3$ & $13 \cdot 9$ & $13 \cdot 7$ & $13 \cdot 3$ & $17 \cdot 1$ & $15 \cdot 7$ & $0 \cdot 2$ & - & 86.9 & 88.0 & $83 \cdot 0$ & $81 \cdot 4$ & $84 \cdot 2$ & 7 & $84 \cdot 8$ & $85 \cdot 7$ & $85 \cdot 6$ & $4 \cdot 2$ & $84 \cdot 4$ & $84 \cdot 2$ & $84 \cdot 2$ & $84 \cdot 2$ \\
\hline Treponema bryantii & 15.5 & $16 \cdot 4$ & $18 \cdot 8$ & $16 \cdot 3$ & $16 \cdot 5$ & $14 \cdot 9$ & $16 \cdot 2$ & 14.9 & 14.4 & 14.4 & - & $88 \cdot 1$ & 83.8 & $84 \cdot 7$ & $86 \cdot 4$ & & 86.8 & $87 \cdot 3$ & $87 \cdot 3$ & $4 \cdot 6$ & $84 \cdot 6$ & $84 \cdot 4$ & 84.9 & $84 \cdot 9$ \\
\hline Treponema pectinovorum & $15 \cdot 7$ & $15 \cdot 5$ & $18 \cdot 5$ & $15 \cdot 6$ & $16 \cdot 2$ & $15 \cdot 1$ & 17.5 & $16 \cdot 3$ & $13 \cdot 3$ & $13 \cdot 1$ & $13 \cdot 0$ & - & $86 \cdot 3$ & 83.8 & 86.9 & 88.9 & 88 & $88 \cdot 3$ & $88 \cdot 5$ & $84 \cdot 8$ & $84 \cdot 7$ & 85.0 & $85 \cdot 1$ & $84 \cdot 7$ \\
\hline Treponema saccharophilum & 17.8 & $18 \cdot 0$ & $20 \cdot 2$ & 18.5 & $18 \cdot 4$ & $17 \cdot 2$ & $19 \cdot 4$ & $18 \cdot 3$ & $19 \cdot 5$ & $19 \cdot 3$ & $18 \cdot 3$ & $15 \cdot 2$ & - & 82.9 & 88.0 & 83.5 & $83 \cdot 7$ & 83.6 & $83 \cdot 6$ & $81 \cdot 2$ & $81 \cdot 3$ & $81 \cdot 2$ & $81 \cdot 2$ & $81 \cdot 3$ \\
\hline Treponema succinifaciens & $21 \cdot 5$ & $20 \cdot 8$ & $20 \cdot 9$ & $22 \cdot 3$ & $22 \cdot 4$ & $20 \cdot 0$ & $20 \cdot 4$ & $19 \cdot 9$ & 21.0 & $21 \cdot 4$ & $17 \cdot 1$ & $18 \cdot 3$ & $19 \cdot 4$ & - & 87.7 & $84 \cdot 6$ & $85 \cdot 2$ & $85 \cdot 6$ & $85 \cdot 5$ & $84 \cdot 1$ & $84 \cdot 0$ & 83.8 & $84 \cdot 4$ & $84 \cdot 2$ \\
\hline Treponema sp. CA & $18 \cdot 0$ & $18 \cdot 5$ & $18 \cdot 5$ & 18.6 & 18.6 & 17.5 & $16 \cdot 1$ & $16 \cdot 5$ & $17 \cdot 7$ & 17.7 & $15 \cdot 0$ & $14 \cdot 4$ & $13 \cdot 0$ & $13 \cdot 5$ & - & $86 \cdot 2$ & $85 \cdot 5$ & 85.9 & $85 \cdot 8$ & $85 \cdot 5$ & $85 \cdot 5$ & $85 \cdot 5$ & $85 \cdot 6$ & $85 \cdot 6$ \\
\hline Treponema Smibert-2 & $14 \cdot 8$ & $15 \cdot 1$ & $17 \cdot 4$ & $15 \cdot 3$ & $15 \cdot 7$ & $14 \cdot 6$ & 17.5 & $17 \cdot 2$ & 13.5 & $13 \cdot 4$ & $12 \cdot 7$ & $12 \cdot 0$ & $18 \cdot 6$ & $17 \cdot 2$ & $15 \cdot 2$ & - & $89 \cdot 3$ & $90 \cdot 2$ & $90 \cdot 3$ & $86 \cdot 3$ & 86.4 & $86 \cdot 5$ & 87.0 & $86 \cdot 8$ \\
\hline Treponema Smibert-3 & 17.8 & $17 \cdot 1$ & 18.5 & $17 \cdot 7$ & 18.2 & $17 \cdot 1$ & $17 \cdot 3$ & 17.6 & 17.2 & $17 \cdot 0$ & $14 \cdot 5$ & $12 \cdot 6$ & $18 \cdot 4$ & 16.5 & $16 \cdot 1$ & $11 \cdot 6$ & - & $98 \cdot 0$ & $98 \cdot 0$ & $86 \cdot 6$ & $86 \cdot 4$ & 86.6 & 86.7 & $86 \cdot 1$ \\
\hline Treponema Smibert-4 & 17.0 & $16 \cdot 5$ & $19 \cdot 1$ & 17.7 & $18 \cdot 0$ & $16 \cdot 8$ & $17 \cdot 9$ & $17 \cdot 7$ & 16.0 & 15.9 & $13 \cdot 9$ & 12.8 & $18 \cdot 5$ & 15.9 & 15.7 & $10 \cdot 4$ & 20 & - & $99 \cdot 9$ & $86 \cdot 2$ & $86 \cdot 1$ & $86 \cdot 1$ & $86 \cdot 1$ & 85.8 \\
\hline Treponema amylovorum & $17 \cdot 1$ & $16 \cdot 7$ & $19 \cdot 2$ & 17.8 & 17.8 & 16.9 & 17.9 & 17.7 & $16 \cdot 1$ & $16 \cdot 0$ & 13.9 & $12 \cdot 5$ & $18 \cdot 5$ & $16 \cdot 1$ & $15 \cdot 7$ & $10 \cdot 3$ & $2 \cdot 0$ & $0 \cdot 1$ & - & $86 \cdot 2$ & 86.0 & $86 \cdot 0$ & $86 \cdot 1$ & 85.7 \\
\hline Treponema Smibert-5 & $18 \cdot 1$ & 17.6 & 19.7 & $18 \cdot 6$ & $18 \cdot 5$ & $18 \cdot 5$ & $18 \cdot 2$ & $17 \cdot 4$ & 17.8 & $17 \cdot 7$ & $17 \cdot 2$ & 17.0 & $21 \cdot 6$ & 17.8 & $16 \cdot 1$ & $15 \cdot 1$ & $14 \cdot 7$ & $15 \cdot 2$ & $15 \cdot 3$ & - & 97.8 & 98.0 & 97.4 & 98.2 \\
\hline $\begin{array}{l}\text { Treponema socranskii } \\
\text { subsp. buccale }\end{array}$ & 18.0 & $17 \cdot 9$ & $20 \cdot 2$ & $19 \cdot 0$ & $19 \cdot 0$ & $19 \cdot 0$ & 18.6 & $17 \cdot 8$ & 17.7 & $17 \cdot 5$ & $17 \cdot 2$ & $17 \cdot 2$ & $21 \cdot 5$ & $18 \cdot 0$ & $16 \cdot 1$ & $15 \cdot 0$ & $15 \cdot 0$ & $15 \cdot 4$ & $15 \cdot 5$ & $2 \cdot 2$ & - & 98.6 & $98 \cdot 3$ & $99 \cdot 3$ \\
\hline $\begin{array}{l}\text { Treponema socranskii } \\
\text { subsp. socranskii }\end{array}$ & $18 \cdot 1$ & $18 \cdot 4$ & $20 \cdot 2$ & $19 \cdot 0$ & $19 \cdot 3$ & $19 \cdot 0$ & 18.7 & 18.0 & 17.9 & $17 \cdot 7$ & $17 \cdot 4$ & $16 \cdot 8$ & $21 \cdot 7$ & 18.2 & $16 \cdot 1$ & 14.9 & 14.8 & $15 \cdot 4$ & $15 \cdot 5$ & 2.0 & 1.4 & - & 98.4 & 98.9 \\
\hline $\begin{array}{l}\text { Treponema socranskii } \\
\text { subsp. paredis }\end{array}$ & 18.7 & $18 \cdot 6$ & 20.7 & 19.6 & 19.7 & $19 \cdot 4$ & 18.9 & 17.8 & 17.9 & 17.7 & $16 \cdot 9$ & 16.6 & $21 \cdot 6$ & 17.5 & 16.0 & $14 \cdot 3$ & 14.6 & $15 \cdot 3$ & 15.4 & 2.7 & 1.7 & 1.6 & - & 98.2 \\
\hline $\begin{array}{l}\text { Treponema socranskii } \\
\text { subsp. } 04\end{array}$ & 17.5 & 17.6 & $19 \cdot 4$ & 18.8 & $19 \cdot 0$ & $18 \cdot 6$ & 18.0 & 18.0 & 18.0 & 17.8 & 16.8 & $17 \cdot 1$ & $21 \cdot 5$ & 17.8 & 15.9 & 14.5 & $15 \cdot 3$ & 15.8 & 15.9 & 1.9 & 0.7 & $1 \cdot 1$ & 1.9 & - \\
\hline
\end{tabular}

characteristics and cellular fatty acid profiles $(25,26$, $27,28,40$ ). In this study, the $16 \mathrm{~S}$ rRNA genes were sequenced for 47 strains that were representative of these groups. If strains were identified definitively from analysis of partial sequences (i.e. approximately 350 500 bases), then full sequences of these strains were not determined. Nearly complete sequences of the $16 \mathrm{~S}$ rRNA genes were determined for 26 strains (Table 2). At least one full sequence for each group has been submitted to GenBank (Table 1). The sequences of all other strains are available from the corresponding author. Many strains from the collection could not be identified because a PCR product of the DNA was not obtained using the primers described above. Preliminary studies using a newly designed, spirochaetalselective forward primer (primer F29; 5 ' CGG YGC GTC TTA AVC ATG CAA 3', E. coli position 40-60) indicated that the sequences of one or more of the original primers used for amplification were not complementary to target sequences of some of the isolates. DNA from these samples is presently being amplified for sequence analysis.

Table 3 shows a similarity matrix that was constructed on the basis of 1445 base position comparisons for $16 \mathrm{~S}$ rRNA sequences of the spirochaetal strains under examination. A phylogenetic tree (Fig. 1) was constructed from the corrected distance matrix using the neighbour-joining method. Based on these analyses, five distinct species were identified from the Smibert collection with the cut-off for separate species designation at about $98 \%$ sequence similarity. These new species are designated as Treponema Smibert-1 through Treponema Smibert-5 (Fig. 1). As described in detail below, two of these species are newly named and three have not yet been characterized. Other strains were identified as previously described species, such as $T$. socranskii, $T$. pectinovorum, $T$. denticola and $T$. vincentii.

Strain D4B-1, the sole isolate of Treponema Smibert-1, was nearly identical in 500 base comparisons to spirochaetal clone NZM3125 (phylotype 17), previously described from clonal analysis of plaque DNA from a patient with severe periodontitis (5). The closest relative of D4B-1 was $T$. pectinovorum at about $88 \%$ sequence similarity. While this work was in progress, this strain was shown to be nearly identical in sequence to the recently described species $T$. maltophilum (48). However, cells of D4B-1 had two (and occasionally three) periplasmic flagella inserted at each end of the cell (Fig. 2), whereas strains of T. maltophilum were reported to have only one flagellum inserted at each end (48). Strain D4B-1 was defined as belonging to cellular fatty acid group $\mathrm{F}$ or $\mathrm{C}$. No clinical data were available for this strain.

Treponema Smibert-2 contained nine strains that possessed identical partial sequences. The closest relatives of Treponema Smibert-2 were Treponema Smibert-3 and Treponema Smibert-4 at about $90 \%$ sequence similarity. Cells measured about $0 \cdot 15-0 \cdot 2 \mu \mathrm{m}$ by $3-5 \mu \mathrm{m}$ and had a single periplasmic flagellum inserted at each end of the cell. Smibert-2 did not 


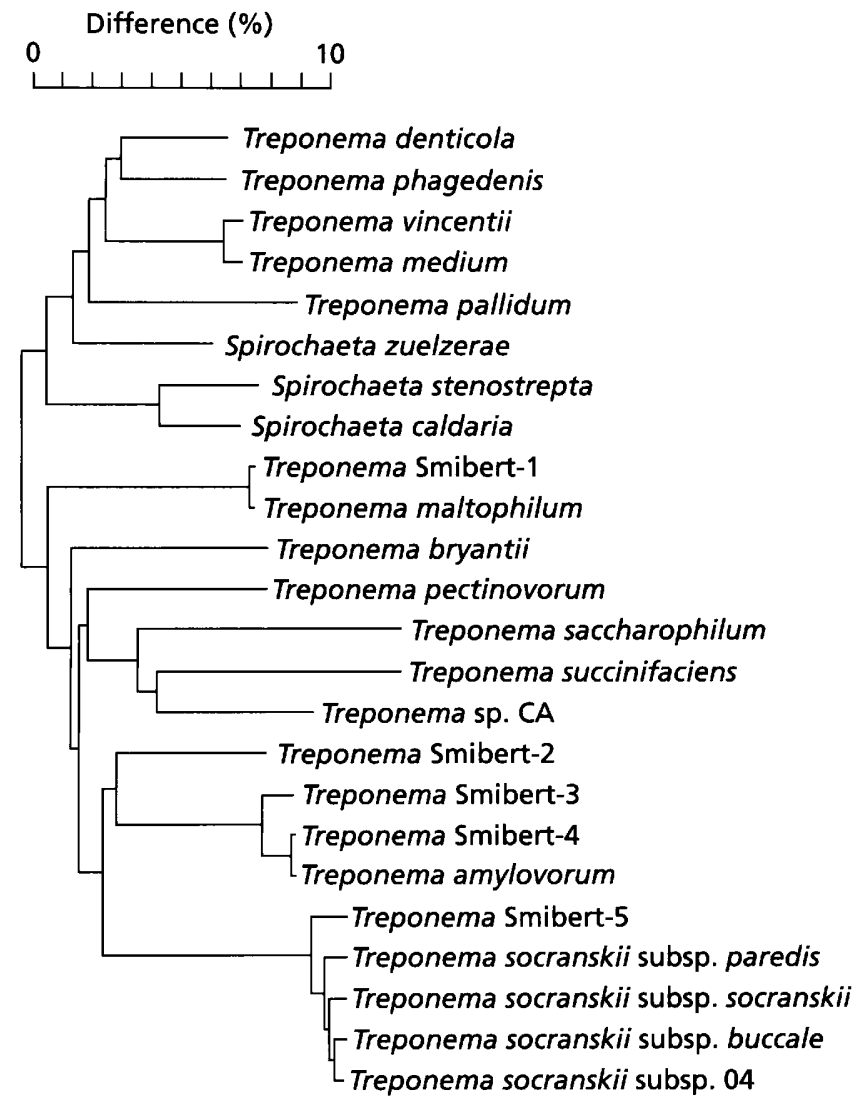

Fig. 1. Phylogenetic tree of the spirochaetes illustrating the phylogenetic positions of oral cultivable species of Treponema. The tree was constructed from comparisons of sequences of the specific strains listed in Table 1. T. vincentii strain N9 (not ATCC 35580) was used for the analysis. The scale bar represents a $10 \%$ difference in nucleotide sequence as determined by taking the sum of all of the horizontal lines connecting two species.

match with any of the phylotypes previously described from clonal analysis of plaque DNA (5). These isolates were previously described as belonging to six separate cellular fatty acid groups - T. socranskii subsp. socranskii, E, F, S, T and U. Strains of this new species were isolated from supragingival and subgingival plaque from healthy and diseased sites (Table 2).

Treponema Smibert-3 was represented by a single strain, D36ER-1, and was identical in 500 base comparisons to spirochaetal clone NZM3109 (cluster 20) previously described from clonal analysis of plaque DNA (5). Treponema Smibert-3 was identified previously as belonging to cellular fatty acid group $\mathrm{F}$. As described below, other strains belonging to cellular fatty acid group $\mathrm{F}$ were identified as Treponema Smibert-2 (Table 2). Cells of Treponema Smibert-3 measured about $0.2 \mu \mathrm{m}$ by $3-5 \mu \mathrm{m}$ and had four to five periplasmic flagella inserted at each end of the cell. Treponema Smibert-3 was isolated from supragingival plaque from a site characterized as juvenile periodontitis. As shown in Table 3 and Fig. 1, the closest cultivable relative of Treponema Smibert-3 was
Treponema Smibert-4 at about $98 \%$ sequence similarity.

Treponema Smibert-4 was represented by a single strain, D62CR-I2, and differed by two bases in 500 base sequence comparisons to spirochaetal clone NZM3124 (cluster 19) previously described from clonal analysis of plaque DNA (5). While this work was in progress, this strain was shown to be identical in sequence to the recently described species $T$. amylovorum (49). Consequently, strain D62CR-I2 represents the second isolate of $T$. amylovorum. As with the type strain of T. amylovorum, strain D62CRI2 was also isolated from plaque from a site characterized as severe periodontitis. Smibert- 4 was identified previously as belonging to cellular fatty acid group $\mathrm{N}$ (Table 2). Other strains of cellular fatty acid group $\mathrm{N}$ were not related to Treponema Smibert-2 (Table 2).

Treponema Smibert-5 was represented by a single strain, D120CR-1, which was identified from cellular fatty acid analysis as $T$. socranskii subsp. buccale. However, Treponema Smibert-5 probably represents a new species that is closely related to the three subspecies of $T$. socranskii each at about $98 \%$ sequence similarity (Fig. 1). DNA-DNA reassociation experiments will be necessary to determine if separate species (or subspecies) designation of Treponema Smibert-5 is warranted. Cells of Smibert- 5 measured about $0 \cdot 2 \mu \mathrm{m}$ by $3-5 \mu \mathrm{m}$ and had one periplasmic flagellum inserted at each end of the cell. This ultrastructure is similar to that of cells of subspecies of $T$. socranskii. Treponema Smibert- 5 was isolated from subgingival plaque from a healthy site.

Strains belonging to cellular fatty acid groups C, DB, E, some F, G, L, some $N$ and V were found to be nearly identical to subspecies of $T$. socranskii and did not represent new species (Table 2). The type strains of subspecies of $T$. socranskii-subspp. socranskii, buccale and paredis - formed three separate phylogenetic branches with intragenic sequence similarities of over $98 \%$ (Table 2, Fig. 1). Preliminary studies have shown that at least five members of a fourth subspecies of $T$. socranskii (referred to as subsp. 04) cluster together to form a separate fourth branch (Table 2). However, to determine if each subspecies is a phylogenetically coherent group, comparisons of complete 16S rRNA gene sequences for multiple strains of each subspecies will be necessary.

The two strains of $T$. vincentii that were sequenced had nearly identical sequences, differing by four bases. The closest relative of $T$. vincentii was T. medium at $98.7 \%$ sequence similarity. However, the levels of DNADNA homology between these species was reported to be $28 \%$ (46), thus warranting separate species designation. Both $T$. vincentii and $T$. medium had about $92 \%$ sequence similarity to $T$. denticola and Treponema phagedenis (Table 3, Fig. 1).

We previously reported that 16S rRNA sequences of some spirochaetes (including the treponemes) possess 


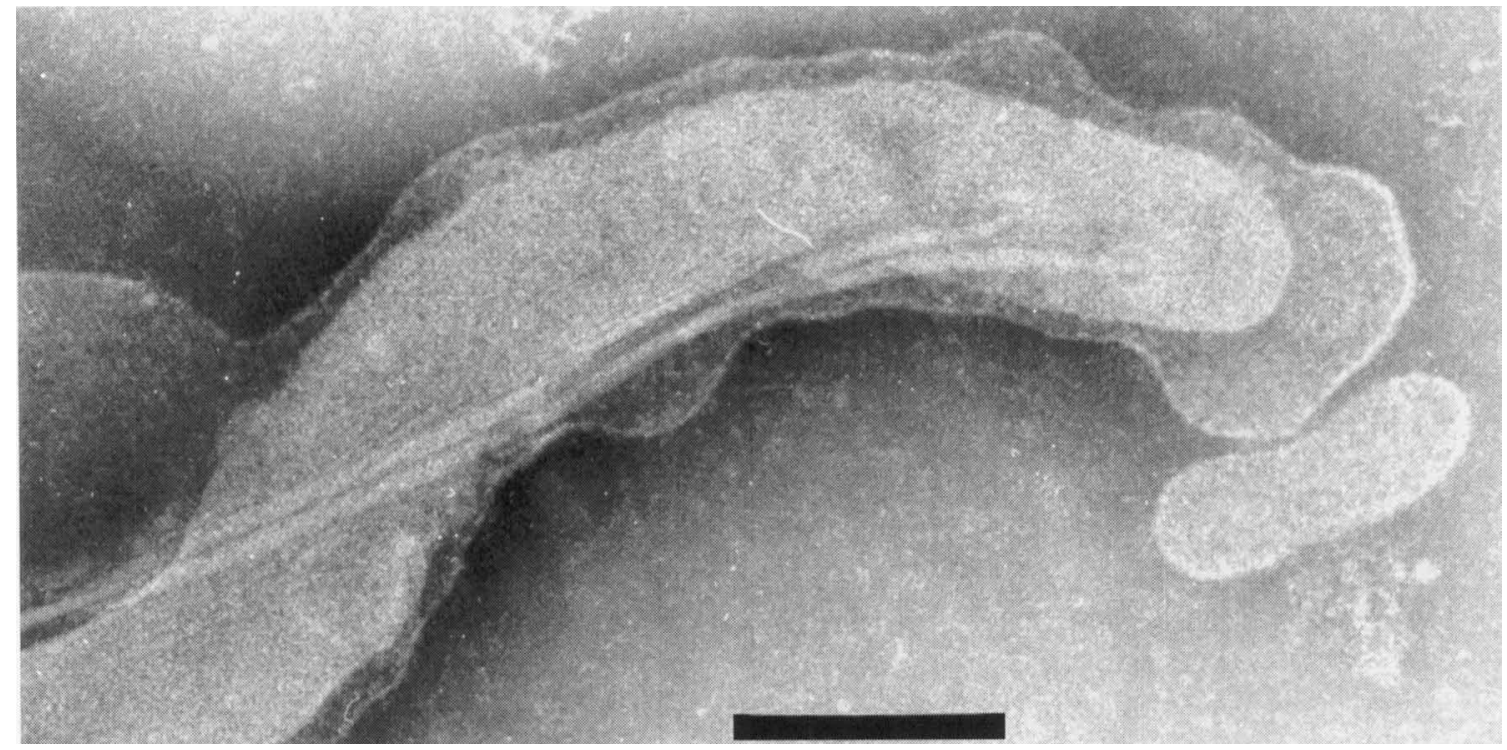

Fig. 2. Transmission electron micrograph of a negatively stained cell of strain D4B-1 (Treponema sp. Smibert-1), which is phylogenetically identical to $T$. maltophilum. Two periplasmic flagella are inserted at the end of the cell. Cells of $T$. maltophilum were reported to have only one flagellum at each end (48). Bar, $0.2 \mu \mathrm{m}$.

a highly variable $5^{\prime}$ extension of $20-40$ bases (31). These extensions, which are unique among prokaryotes and eukaryotes, were determined by directly sequencing $16 \mathrm{~S}$ rRNA using reverse transcriptase (31). The rRNA sequences of $T$. vincentii ATCC 35580 and T. socranskii subsp. socranskii ATCC 35536 were determined using this methodology and they did possess unique $5^{\prime}$ extensions. It is not known if the other strains examined possess a $5^{\prime}$ extension. The present PCR methodology utilizes a forward primer that begins at position 8 , and thus the $5^{\prime}$ extension would not be detected.

Historic reference strains Treponema oralis (no strain designation), TRRD, MRB, Fuji, IPP, Jethro and T32A were identified as strains of $T$. denticola. There were four or fewer base differences in the sequences of any historic strain as compared to the sequence of $T$. denticola ATCC 33520. Strains of $T$. oralis were previously reported to have a flagellar arrangement of 1-2-1 (42); however the strain available from the Smibert collection had a 2-4-2 arrangement.

Four of the historic strains (Fuji, IPP, Jethro and T32A) and three of the Smibert isolates [D156DR-4, D113DR-1 and D46CPE-1 (type species of $T$. socranskii subsp. paredis)] were contaminated with Mycoplasma orale as deduced from sequence analysis. The presence of mycoplasmas is not surprising since treponemes were cultured on medium supplemented with filter-sterilized bovine serum. Mycoplasmas are able to pass through a $0.22 \mu \mathrm{m}$ filter. Mixed cultures of organisms are immediately evident by $16 \mathrm{~S}$ rRNA sequencing as double reads on the sequencing gels. If the sequence for one of the species is known in a binary mixture, then the sequence of the second can be determined and the organism can be placed phylogenetically. It is unlikely that our laboratory is the source of the contamination since DNA was extracted directly from the frozen samples.

Cellular fatty acid analysis can be used to reliably differentiate many bacterial species and is considered a useful taxonomic tool (24). However, the grouping of human oral treponemes by cellular fatty acid analysis did not always correlate with $16 \mathrm{~S}$ rRNA gene sequence comparative analysis (Table 2). As described above, six cellular fatty acid groups are represented in the phylogenetically coherent Treponema Smibert-2. Cellular fatty acid groups C, E, G, L, V, F, DB and one strain of $\mathrm{N}$ were closely related to subspecies of $T$. socranskii (Table 2). One strain (D62CR-I2) of group $\mathrm{N}$ formed the new species Treponema Smibert-4, whereas another strain (D46DR-I1) of cellular fatty acid group $\mathrm{N}$ was identified as $T$. denticola. Strain D36ER-1 and strain D79VR-1 of cellular fatty acid group F were identified as Treponema Smibert-3 and T. socranskii subsp. socranskii, respectively, whereas most strains of cellular fatty acid group $\mathrm{F}$ belonged to the new species Treponema Smibert-2. One possibility for these discrepancies is that cellular fatty acid compositions of micro-organisms vary with growth media, age of the culture and the temperature used for incubation (24). Cells were initially grown in media containing serum, which contains fatty acids that cannot be washed easily from cells. Efforts were made to remove these fatty acids by transferring cells into medium without fatty acids for $24 \mathrm{~h}$ and then transferring cells to standardized media for $2 \mathrm{~h}$. Since 
Treponema pallidum, Borrelia burgdorferi and some oral treponemes are capable of only limited synthesis of cellular fatty acids, they incorporate fatty acids into their lipids relatively nonselectively from their environment $(2,46)$. If this requirement for fatty acids was true for most oral treponemes, then cellular fatty acid analysis would reflect the fatty acids present in the culture medium rather then from synthesis of fatty acids by the organism.

Whilst many species of oral treponemes have not yet been cultivated, Dr Smibert had isolated at least five novel species. 16S rRNA sequence analysis has allowed the unambiguous identification and phylogenetic placement of each of these species. Three of these new species (Treponema Smibert-1, Treponema Smibert-3 and Treponema Smibert-4) corresponded to species postulated to exist based upon 16S rRNA analysis of clones obtained from PCR amplification of DNA from plaque of a patient with severe periodontal disease (5). Consequently, there are now at least ten cultivable oral species of Treponema.

To formally name the three novel species reported in this study, a detailed description of phenotypic and other characteristics of strains is necessary. Furthermore, two of the species (Smibert-3 and Smibert 5) are each represented by only a single isolate. Formal naming of these latter two species should be avoided until additional isolates are obtained and characterized. Furthermore, a cut-off value of about $98 \%$ sequence similarity was used to differentiate species of oral treponemes. At this cut-off level, it is suggested that DNA-DNA reassociation experiments still be used to confirm separate species designation.

\section{ACKNOWLEDGEMENTS}

We are grateful to Polly Cooper, Dr R. Smibert, Dr W. E. C. Moore and Dr L. V. Moore for providing us with the spirochaete collection and pertinent biochemical and clinical data for each of the strains.

This work was supported in part by Public Health Service grants DE-10374 and DE-11443 from the National Institute of Dental Research.

\section{REFERENCES}

1. Armitage, G. C., Dickison, W. R., Jenderseck, R. S., Levine, S. M. \& Chambers, D. W. (1982). Relationship between the percentage of subgingival spirochetes and severity of periodontal disease. $J$ Periodontol 53, 550-556.

2. Belisle, J. T., Brandt, M. E., Radolf, J. D. \& Norgard, M. V. (1994). Fatty acids of Treponema pallidum and Borrelia burgdorferi lipoproteins. J Bacteriol 176, 2151-2157.

3. Carranza, F. A., Saglie, R., Newman, M. G. \& Valentin, P. L. (1983). Scanning and transmission electron microscopic study of tissue invading microorganisms in localized juvenile periodontitis. J Periodontal Res 54, 598-617.

4. Choi, B.-K., Nattermann, H., Grund, S., Haider, W. \& Göbel, U. B. (1997). Spirochetes from digital dermatitis lesions in cattle are closely related to treponemes associated with human periodontitis. Int J Syst Bacteriol 47, 175-181.
5. Choi, B.-K., Paster, B. J., Dewhirst, F. E. \& Göbel, U. B. (1994). Diversity of cultivable and uncultivable oral spirochetes from a patient with severe destructive periodontitis. Infect Immun 62, 1889-1895.

6. Dahle, U. R., Tronstad, L. \& Olsen, I. (1993). Spirochaetes in oral infections. Endod Dent Traumatol 9, 87-94.

7. Defosse, D. L., Johnson, R. C., Paster, B. J., Dewhirst, F. E. \& Fraser, G. J. (1995). Brevinema andersonii gen. nov., sp. nov., an infectious spirochete isolated from the short-tailed shrew (Blarina brevicauda) and the white-footed mouse (Peromyscus leucopus). Int J Syst Bacteriol 45, 78-84.

8. Falkler, W. A., Jr, Martin, S. A., Vincent, J. W., Tall, B. D., Nauman, R. K. \& Suzuki, J. B. (1987). A clinical, demographic and microbiologic study of ANUG patients in an urban dental school. J Clin Periodontol 14, 307-314.

9. Fiehn, N.-E. (1989). Small-sized oral spirochetes and periodontal disease. APMIS 97(suppl. 7), 1-31.

10. Fox, J. G., Yan, L., Dewhirst, F. E., Paster, B. J., Murphy, J. C., Hayward, A., Belcher, J. C. \& Mendes, E. N. (1995). Helicobacter bilis sp. nov., a novel Helicobacter isolated from bile, livers, and intestines of aged, inbred mouse strains. $J$ Clin Microbiol 33, 445-454.

11. Frank, R. M. (1980). Bacterial penetration in the apical pocket wall of advanced human periodontitis. $J$ Periodontal Res 15, 563-570.

12. Fraser, G. J., Paster, B. J., Belcher, J. C. \& Dewhirst, F. E. (1995). Phylogeny of cultivable oral treponemes from the Smibert collection. J Dent Res 74, 249.

13. Hookey, J. V., Barrett, S. P., Reed, C. S. \& Barber, P. (1994). Phylogeny of human intestinal spirochaetes inferred from $16 \mathrm{~S}$ rDNA sequence comparisons. FEMS Microbiol Lett 117, 345-350.

14. Johnson, B. D. \& Engel, D. (1986). Acute necrotizing ulcerative gingivitis: a review of diagnosis, etiology and treatment. J Periodontol 57, 141-150.

15. Jukes, T. H. \& Cantor, C. R. (1969). Evolution of protein molecules. In Mammalian Protein Metabolism, vol. 3, pp. 21-132. Edited by H. N. Munro. New York: Academic Press.

16. Keyes, P. H., Wright, W. E. \& Howard, S. A. (1978). The use of phase-contrast microscopy and chemotherapy in the diagnosis and treatment of periodontal lesions - an initial report. Quint Int 1, 51-56.

17. Leschine, S. B. \& Canale-Parola, E. (1980). Rifampin as a selective agent for isolation of oral spirochetes. $J$ Clin Microbiol 12, 792-795.

18. Listgarten, M. A. (1965). Electron microscopic observation on the bacterial flora of acute necrotizing ulcerative gingivitis. J Periodontol 36, 328-339.

19. Listgarten, M. A. (1976). Structure of the microbial flora associated with periodontal health and disease in man. A light and electron microscopic study. $J$ Periodontol 47, $1-18$.

20. Listgarten, M. A. \& Hellden, L. (1978). Relative distribution of bacteria at clinically healthy and periodontally diseased sites in humans. J Clin Periodontol 5, 115-132.

21. Loesche, W. J. (1988). The role of spirochetes in periodontal disease. Adv Dent Res 2, 275-283.

22. Maidak, B. L., Olsen, G. J., Larsen, N., Overbeek, R., McCaughey, M. J. \& Woese, C.R. (1997). The RDP (Ribosomal Database Project). Nucleic Acids Res 25, 109-111. 
23. Mikx, F. H. M., Matee, M. L. \& Maltha, J. C. (1986). The occurrence of oral spirochetes in relation to age and periodontal disease. In Borderland Between Caries and Periodontal Disease, pp. 391-399. Edited by T. Lehner \& G. Cimasoni. Geneva: Editions Medicine et Hygiene.

24. Moore, L. V. H., Bourne, D. M. \& Moore, W. E. C. (1994). Comparative distribution and taxonomic value of cellular fatty acids in thirty-three genera of anaerobic gram-negative bacilli. Int J Syst Bacteriol 44, 338-347.

25. Moore, L. V., Moore, W. E. C., Cato, E. P., Smibert, R. M., Burmeister, J. A., Best, A. M. \& Ranney, R. R. (1987). Bacteriology of human gingivitis. J Dent Res 66, 989-995.

26. Moore, L. V. H., Moore, W. E. C., Riley, C., Brooks, C. N., Burmeister, J. A. \& Smibert, R. M. (1993). Periodontal microflora of HIV positive subjects with gingivitis or adult periodontitis. $J$ Periodontol 64, 48-56.

27. Moore, W. E. C., Moore, L. V., Ranney, R. R., Smibert, R. M., Burmeister, J. A. \& Schenkein, H. A. (1991). The microflora of periodontal sites showing active destructive progression. $J$ Clin Periodontol 18, 729-739.

28. Moore, W. E. C., Moore, L. V., Cato, E. P., Smibert, R. M., Burmeister, J. A., Pascanis, K. G. \& Ranney, R. R. (1985). Comparative bacteriology of juvenile periodontitis. Infect Immun 48, 507-519.

29. Muller, H.-P. \& Flores de Jacoby, L. (1987). Distribution of morphologically different microorganisms associated with active periodontal lesions. J Clin Periodontol 14, 110-117.

30. Paster, B. J. \& Dewhirst, F. E. (1988). Phylogeny of campylobacters, wolinellas, Bacteroides gracilis and Bacteroides ureolyticus by $16 \mathrm{~S}$ ribosomal ribonucleic acid sequencing. Int J Syst Bacteriol 38, 56-62.

31. Paster, B. J., Dewhirst, F. E., Weisburg, W. G. \& 7 other authors (1991). Phylogenetic analysis of the spirochetes. $J$ Bacteriol 173, 6101-6109.

32. Qiu, Y.-S., Klitorinos, A., Rahal, M. D., Siboo, R. \& Chan, E. C. S. (1994). Enumeration of viable oral spirochetes from periodontal pockets. Oral Microbiol Immunol 9, 301-304.

33. Riviere, G. R., Weisz, K. S., Adams, D. F. \& Thomas, D. D. (1991). Pathogen-related oral spirochetes from dental plaque are invasive. Infect Immun 59, 3377-3380.

34. Riviere, G. R., Weisz, K. S., Simonson, L. G. \& Lukehart, S. A. (1991). Pathogen-related spirochetes identified within gingival tissue from patients with acute necrotizing ulcerative gingivitis. Infect Immun 59, 2653-2657.

35. Rosenstein, D. I., Riviere, G. R. \& Elott, K. S. (1993). HIVassociated periodontal disease: new oral spirochete found. $J A D A$ 124, 76-80.

36. Saglie, R., Newman, M. G., Carranza, F. A., Jr \& Pattison, G. L. (1982). Bacterial invasion of gingiva in advanced periodontitis in humans. J Periodontol 53, 217-222.
37. Saitou, N. \& Nei, M. (1987). The neighbor-joining method: a new method for reconstructing phylogenetic trees. Mol Biol Evol 4, 406-425.

38. Simonson, L. G., Goodman, C., Bial, J. \& Morton, H. E. (1988). Quantitative relationship of Treponema denticola to severity of periodontal disease. Infect Immun 56, 726-728.

39. Smibert, R. M. (1984). Genus III. Treponema Schaudinn $1905,1728^{\mathrm{AL}}$. In Bergey's Manual of Systematic Bacteriology, vol. 1, pp. 49-57. Edited by N. R. Krieg \& J. G. Holt. Baltimore: Williams \& Wilkins.

40. Smibert, R. M. \& Burmeister, J. A. (1983). Treponema pectinovorum $\mathrm{sp}$. nov. isolated from humans with periodontitis. Int J Syst Bacteriol 33, 852-856.

41. Smibert, R. M., Johnson, J. L. \& Ranney, R. R. (1984). Treponema socranskii sp. nov., Treponema socranskii subsp. socranskii subsp. nov., Treponema socranskii subsp. buccale subsp. nov., Treponema socranskii subsp. paredis subsp. nov. isolated from the human periodontia. Int $J$ Syst Bacteriol 34, 457-462.

42. Socransky, S. S., Listgarten, M. A., Hubersak, C., Cotmore, J. \& Clark, A. (1969). Morphological and biochemical differentiation of three types of small oral spirochetes. $J$ Bacteriol 98, 878-882.

43. Trope, M., Rosenberg, E. S. \& Tronstad, L. (1992). Darkfield microscopic spirochete count in the differentiation of endodontic and periodontal abscesses. $J$ Endodont 18 , 82-86.

44. Umeda, M., Ishikawa, I., Benno, Y. \& Mitsuoka, T. (1990). Improved detection of oral spirochetes in an anaerobic culture method. Oral Microbiol Immunol 5, 90-94.

45. Umemoto, T., Nakazawa, F., Hoshino, E., Okada, K., Fukunaga, M. \& Namikawa, I. (1997). Treponema medium $\mathrm{sp.}$ nov., isolated from human subgingival dental plaque. Int $J$ Syst Bacteriol 47, 67-72.

46. Van Horn, K. G. \& Smibert, R. M. (1982). Fatty acid requirement of Treponema denticola and Treponema vincentii. Can J Microbiol 28, 344-350.

47. Westergaard, J. \& Fiehn, N.-E. (1987). Morphological distribution of spirochetes in subgingival plaque from advanced marginal periodontitis in humans. APMIS Sect $B$ Microbiol 95, 49-55.

48. Wyss, C., Choi, B. K., Schüpbach, P., Guggenheim, B. \& Göbel, U. B. (1996). Treponema maltophilum sp. nov., a small oral spirochete isolated from human periodontal lesions. Int $J$ Syst Bacteriol 46, 745-752.

49. Wyss, C., Choi, B. K., Schüpbach, P., Guggenheim, B. \& Göbel U. B. (1997). Treponema amylovorum sp. nov., a saccharolytic spirochete of medium size isolated from an advanced human periodontal lesion. Int J Syst Bacteriol 47, 842-845. 